\title{
A Novel Optimal Shunt Capacitors Placement and Sizing Technique for Cost Minimization
}

This paper was downloaded from TechRxiv (https://www.techrxiv.org).

LICENSE

CC BY-SA 4.0

SUBMISSION DATE / POSTED DATE

$11-01-2022$ / 14-01-2022

CITATION

Mtonga, Thomson; Kaberere, Keren K.; Irungu, George Kimani (2022): A Novel Optimal Shunt Capacitors Placement and Sizing Technique for Cost Minimization. TechRxiv. Preprint.

https://doi.org/10.36227/techrxiv.18141971.v1

$\mathrm{DOI}$

10.36227/techrxiv.18141971.v1 


\title{
A Novel Optimal Shunt Capacitors Placement and Sizing Technique for Cost Minimization
}

\author{
Thomson P. M. Mtonga, Keren K. Kaberere, and George K. Irungu
}

\begin{abstract}
The installation of shunt capacitors in radial distribution systems leads to reduced branch power flows, branch currents, branch power losses and voltage drops. Consequently, this results in improved voltage profiles and voltage stability margins. However, for efficient attainment of the stated benefits, the shunt capacitors ought to be installed in an optimal manner, that is, optimally sized shunt capacitors need to be installed at the optimum buses of an electrical system. This article proposes a novel approach for optimizing the placement and sizing of shunt capacitors in radial distribution systems with a focus on minimizing the cost of active power losses and shunt capacitors' purchase, installation, operation and maintenance. To reduce the search space, hence the computation time, the proposed approach starts the search process by arranging the buses of the radial distribution system under consideration in pairs. Thereafter, these pairs influence each other to determine the optimum total number of buses to be compensated. The proposed approach was tested on the 34- and 85-bus radial distribution systems and when the simulation results were compared with those obtained by other approaches, it was established that the developed approach was a better option because it gave the least cost.
\end{abstract}

Index Terms-- Active power losses, distribution systems, power flows, search space, shunt capacitors.

\section{INTRODUCTION}

$\mathrm{P}$ ower system voltages, frequency and power flows (active and reactive) are some of the parameters that power system operators carefully monitor and control. These parameters are monitored to ensure that they stay within prescribed limits and any deviations or pending deviations are dealt with by the implementation of manual (local or remote) or automatic control activities. The switching on and off of shunt capacitors in radial distribution systems is one of the control activities that are either manually or automatically carried out to control power flows and consequently the power system voltages [1], [2].

Shunt capacitors are optimally placed in radial distribution systems to meet the different objectives for which they are installed. A review of some of the existing literature on radial distribution systems' optimal shunt capacitors' placement and sizing for cost minimization shows that a number of

Thomson P. M. Mtonga is with the Department of Civil Aviation, Ministry of Transport and Public Infrastructure, Lilongwe, Malawi (E-mail: mactoppie@yahoo.co.uk).

Keren K. Kaberere ${ }^{1}$ and George K. Irungu ${ }^{2}$ are with the Department of Electrical and Electronic Engineering, Jomo Kenyatta University of Agriculture and Technology (JKUAT), Nairobi, Kenya (E-mail ${ }^{1}$ : kkanuthu@eng.jkuat.ac.ke; E-mail²: girungu@jkuat.ac.ke). researchers have used either of two general approaches to optimize the placement and sizing of shunt capacitors. In the first approach, when researchers want to optimize a given cost function they use different optimization algorithms to determine the optimum shunt capacitor sizes, the optimum total number of buses to be compensated and the specific optimum buses on which to install the optimally sized shunt capacitors [3]-[5]. For example, when this approach is implemented, it may determine that it costs $x$ dollars to have optimally sized shunt capacitors installed at a total of $y$ buses. In case an electric power utility company fails to secure the $x$ dollars, it may have to implement an option with lesser investment costs. This may mean installing optimally sized shunt capacitors at less than $y$ buses and that is where the second approach becomes applicable. In the second approach, when researchers want to optimize a given cost function they specify the total number of buses to be compensated and optimization algorithms are only used to determine the optimum shunt capacitor sizes and the specific optimum buses on which to install the optimally sized shunt capacitors [6]-[8].

In this article the authors propose and evaluate a novel approach for optimizing the placement and sizing of shunt capacitors in radial distribution systems with a focus on minimizing the cost of active power losses and shunt capacitors' purchase, installation, operation and maintenance. In the proposed approach, Multiverse Optimization (MVO) algorithm was used to determine the optimum shunt capacitor sizes, the optimum total number of buses to be compensated and the specific optimum buses on which to install optimally sized shunt capacitors.

The rest of the paper is organized as follows: Section II gives a review of loss minimization through the installation of shunt capacitors while Section III discusses the methodology that was adopted when implementing this work. Thereafter, Section IV briefly discusses the test cases that have been used in this study. Section V presents and discusses the simulation results which are then followed by a conclusion of the article as given in Section VI.

\section{LOSS MINIMIZATION THROUGH THE INSTALLATION OF SHUNT CAPACITORS}

Proper shunt capacitors' placement and sizing helps in reducing the current flowing between two buses. Consequently, this reduces the power system losses and improves an electric power system's voltage profile and voltage stability margin [9]. However, as put forth by Kothari 
and Nagrath [10], the effectiveness of the installed shunt capacitors is so much dependent on the proper selection of their size, ratings and installation buses. The following discussion illustrates the impact of shunt capacitors on both active and reactive power losses as their location is varied.

\section{A. Base Case: Operation without Shunt Capacitors}

Fig. 1 gives a schematic representation of a radial feeder spanning between two areas. Bus-1 is at the feeder's sending end while Bus-2 is at the receiving end.

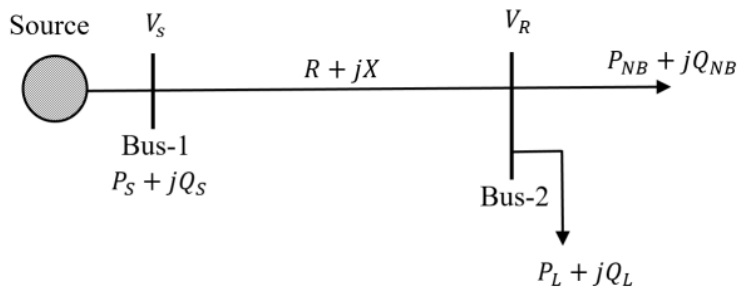

Fig. 1. Operation without shunt capacitors.

With reference to Fig. 1, the line or feeder's active and reactive power losses, $P_{\text {loss }}$ and $Q_{\text {loss }}$ respectively, are given as

$$
\begin{gathered}
P_{\text {loss }}=R \times\left(\frac{P_{S}^{2}+Q_{S}^{2}}{\left|V_{S}\right|^{2}}\right)=R \times\left(\frac{\left(P_{L}+P_{N B}\right)^{2}+\left(Q_{L}+Q_{N B}\right)^{2}}{\left|V_{R}\right|^{2}}\right) \\
Q_{\text {loss }}=X \times\left(\frac{P_{S}^{2}+Q_{S}^{2}}{\left|V_{S}\right|^{2}}\right)=X \times\left(\frac{\left(P_{L}+P_{N B}\right)^{2}+\left(Q_{L}+Q_{N B}\right)^{2}}{\left|V_{R}\right|^{2}}\right)
\end{gathered}
$$

where $P_{S}$ is the per phase sending end active power, $Q_{S}$ is the per phase sending end reactive power, $\left|V_{S}\right|$ is the per phase magnitude of the sending end bus voltage, $P_{L}$ is the per phase active power load at the receiving end bus, $Q_{L}$ is the per phase reactive power load at the receiving end bus, $P_{N B}$ is the summation of the per phase active power loads and losses beyond Bus-2, $Q_{N B}$ is the summation of the per phase reactive power loads and losses beyond Bus-2, $\left|V_{R}\right|$ is the per phase magnitude of the receiving end bus voltage, $R$ and $X$ are the distribution line's resistance and reactance per phase.

For this case, all the demanded active and reactive power is taken from the source.

\section{B. Operation with Shunt Capacitors at Source Side}

Fig. 2 replicates Fig. 1 and illustrates the operation with shunt capacitors installed at the source side.

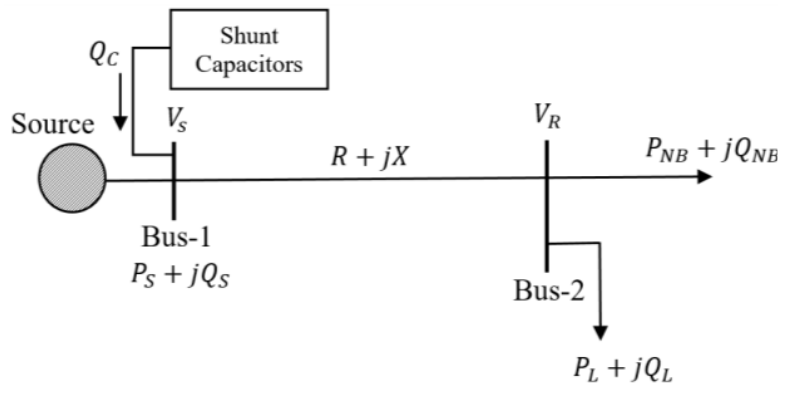

Fig. 2. Operation with shunt capacitors at the source side.

For this case, the active and reactive power losses are the same as those given in (1) and (2), that is, the installation of shunt capacitors at the source side does not help in reducing the electric power system losses occurring between Bus-1 and Bus-2. This is so because the amount of reactive power flowing between Bus-1 and Bus-2 is the same as given for case one. However, for this case

$$
P_{S}+j Q_{S}=P_{S}+j\left(Q_{S}^{\prime}+Q_{C}\right)
$$

where $Q_{C}$ is the reactive power being supplied by the shunt capacitors, and $Q^{\prime}{ }_{S}$ is the reduced amount of reactive power that the source still supplies.

Despite the resulting inability to reduce the radial feeder's electric power losses, the installation of shunt capacitors at the source helps in the reduction of thermal overloads on the source, be it a transformer or a generator [11]. This is so because the installation of shunt capacitors at the source helps with the reduction in the amount of reactive power that the source supplies. This reduction then leads to a consequent reduction in current (which lead to thermal overloads when at high levels) injection from the source. Further, the reduction in current injection from the source to the feeder results in a release of the source's kVA capability [2], [11].

\section{Operation with Shunt Capacitors at the Load Side}

Fig. 3 illustrates the same system but then with the inclusion of shunt capacitors at the load side.

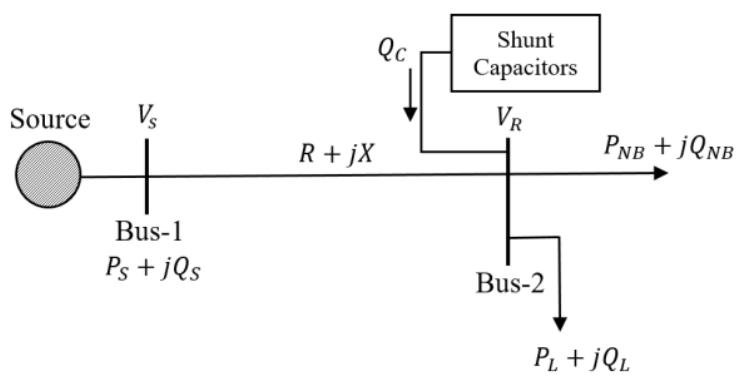

Fig. 3. Operation with shunt capacitors at the load side.

For this case the active and reactive power losses are given as

$$
\begin{aligned}
P_{\text {loss }} & =R \times \frac{\left(P_{S}-\Delta P_{\text {loss }}\right)^{2}+\left(Q_{S}-\Delta Q_{\text {loss }}-Q_{C}\right)^{2}}{\left(\left|V_{S}+\Delta V_{S}\right|\right)^{2}} \\
& =R \times\left(\frac{\left(P_{L}+P_{N B}\right)^{2}+\left(Q_{L}+Q_{N B}-Q_{C}\right)^{2}}{\left|V_{R}\right|^{2}}\right) \\
Q_{\text {loss }} & =X \times\left(\frac{\left(P_{S}-\Delta P_{\text {loss }}\right)^{2}+\left(Q_{S}-\Delta Q_{\text {loss }}-Q_{C}\right)^{2}}{\left(\left|V_{S}+\Delta V_{S}\right|\right)^{2}}\right) \\
& =X \times\left(\frac{\left(P_{L}+P_{N B}\right)^{2}+\left(Q_{L}+Q_{N B}-Q_{C}\right)^{2}}{\left|V_{R}\right|^{2}}\right)
\end{aligned}
$$

where $P_{S}$ is the per phase sending end active power before installation of the shunt capacitors, $Q_{S}$ is the per phase sending end reactive power before installation of the shunt capacitors, $V_{S}$ is the per phase sending end voltage before installation of the shunt capacitors, $\Delta P_{\text {loss }}$ is the per phase change in active power losses between the sending and receiving end buses after installation of the shunt capacitors, $\Delta Q_{\text {loss }}$ is the per 
phase change in reactive power losses between the sending and receiving end buses after installation of the shunt capacitors, and $\Delta V_{s}$ is the per phase change in the sending end voltage after installation of the shunt capacitors.

It may be noted, from (4) and (5), that the introduction of shunt capacitors $\left(Q_{C}\right)$ at the load side reduces the net reactive power loading (i.e. $\left(Q_{L}+Q_{N B}-Q_{C}\right)$ hence also reducing the amount of reactive power flowing between buses 1 and 2 . Additionally, since the reduction in the amount of reactive power flowing between buses 1 and 2 results in reduced current flow; therefore, the active and reactive power losses for this case also becomes less than those for the base case and for the case where the power system is operated with shunt capacitors installed at the source side.

It is advisable to provide reactive power compensation (e.g. through the installation of shunt capacitors) closer to the point of use as practically as possible. This is done to avoid the need to distribute reactive power which leads to high electric current flow between buses and consequently, increased electric power system losses [12]. In [1], it is further stated that the placement of reactive power compensation devices is primarily determined by the reason for compensation. For example, as stated under case two, by locating the shunt capacitors at Bus-1 (i.e. the source side), the source's active power generation or transfer capability would be improved. However, the electric power losses would remain the same. So, if the reason for reactive power compensation is loss minimization, the best location for capacitor placement is the load side. On the other hand, if the reason for reactive power compensation is to reduce thermal overloads on the source or to release the source's kVA capability, then the best location for capacitor placement is the source side.

\section{Methodology}

To meet the study's objective, Multiverse Optimization (MVO) algorithm was used to determine the optimal shunt capacitor sizes, the optimal total number of buses to be compensated and the specific optimal buses on which to install optimally sized shunt capacitors in the 34- and 85-bus radial distribution systems. Simulations were carried out in MATLAB using MATPOWER on a computer with an Intel® Core $^{\mathrm{TM}} \mathrm{i} 3-5005 \mathrm{U}, 2 \mathrm{GHz}$ processor. Before using the MVO, the buses of the 34- and 85-bus radial distribution systems were firstly arranged in pairs. Thereafter, the pairs were allowed to influence each other so as to determine the optimal total number of buses to be compensated. The following subsections discusses the formulation of the problem under study (i.e. the optimal shunt capacitors' placement and sizing problem for cost minimization), the MVO and the proposed cost optimization approach.

\section{A. Formulation of the Optimal Shunt Capacitors' Placement and Sizing Problem.}

The optimal shunt capacitors' placement and sizing problem was formulated through the adoption of the objective function and constraints given in [13]. The objective function was formulated so as to minimize the overall cost of total active power losses and shunt capacitors' purchase, installation, operation and maintenance (O\&M). Minimization of the objective function was constrained by the bus voltage limits and the assumed available minimum and maximum shunt capacitor bank sizes. The adopted objective function is given in (6).

minimize \{Total Cost of Active Power Losses + Shunt

Capacitors' Purchase Cost + Shunt Capacitors'

Installation Cost + Shunt Capacitors O\&M Cost $\}$

$$
\begin{gathered}
\operatorname{minimize}\left\{\mathrm{C}_{\mathrm{e}} \sum_{\mathrm{i}=1}^{\mathrm{nb}} \mathrm{P}_{\mathrm{La}}(\mathrm{i}) \mathrm{T}_{\mathrm{i}}+\mathrm{D}\left(\sum_{\mathrm{j}=1}^{\mathrm{ncap}} \mathrm{C}_{\mathrm{cp}} \mathrm{Q}_{\mathrm{cs}}(\mathrm{j})+\right.\right. \\
\left.\left.\mathrm{ncapC}_{\mathrm{ci}}\right)+\sum_{\mathrm{j}=1}^{\mathrm{ncap}} \mathrm{ncapC}_{\mathrm{co}}\right\}
\end{gathered}
$$

where $C_{e}$ is the average electrical energy cost, $P_{L a}(i)$ is the active power loss in branch $i, \mathrm{nb}$ is the total number of branches in an electrical system, $T_{i}$ is the yearly duration of the load level under consideration, $\mathrm{D}$ is the depreciation factor, $C_{c p}$ is the shunt capacitors' purchase cost per $\operatorname{kVAr}, Q_{c s}(j)$ is the size (kVAr) of the shunt capacitors installed at bus $j$, ncap is the total number of buses with shunt capacitors installed, $C_{c i}$ is the shunt capacitors' installation cost per location, and $C_{c o}$ is the shunt capacitors operation and maintenance $(\mathrm{O} \& \mathrm{M})$ cost per year per location.

The optimization of (6) was subjected to the following operational constraints:

(i) Bus voltages must be kept within their lower $\left(V_{n, \min }\right)$ and upper $\left(V_{n, \max }\right)$ limits. $V_{n, \min }$ and $V_{n, \max }$ were equated to 0.9 and 1.1 p.u. respectively [14].

$$
V_{n, \min } \leq\left|V_{n}\right| \leq V_{n, \max }
$$

(ii) The total reactive power injection at each candidate bus must be governed by the assumed minimum $\left(Q_{c n, \min }\right)$ and maximum $\left(Q_{c n, \max }\right)$ shunt capacitor bank sizes. $Q_{c n, \min }$ and $Q_{c n, \max }$ were equated to 50 and $1500 \mathrm{kVAr}$ respectively. These capacitor sizes were assumed to be in multiples of 50 .

$$
Q_{c n, \min } \leq Q_{c n} \leq Q_{c n, \max }
$$

Table I gives the constants that were used in the optimization of (6).

TABLE I

Constants used in the 34- and 85-Bus Radial Distribution Systems

\begin{tabular}{c|l|c}
\hline No. & Parameter & Value \\
\hline 1 & Average electrical energy cost $\left(C_{e}\right)$ & $0.06 \$ / \mathrm{kWh}$ \\
\hline 2 & Yearly duration of load $\left(T_{i}\right)$ & 8760 hours \\
\hline 3 & $\begin{array}{l}\text { Depreciation factor (D) for the 34-bus } \\
\text { radial distribution system }\end{array}$ & 0.1 \\
\hline 4 & $\begin{array}{l}\text { Depreciation factor (D) for the 85-bus } \\
\text { radial distribution system }\end{array}$ & 0.2 \\
\hline 5 & Shunt capacitors' purchase cost $\left(C_{c p}\right)$ & $25 \$ / \mathrm{kVAr}$ \\
\hline 6 & Shunt capacitors' installation cost $\left(C_{c i}\right)$ & $1600 \$ /$ location \\
\hline 7 & $\begin{array}{l}\text { Shunt capacitors operation and } \\
\text { maintenance (O\&M) cost }\left(C_{c o}\right)\end{array}$ & $300 \$ /$ year/location \\
\hline
\end{tabular}

\section{B. Multiverse Optimization Algorithm}

Multiverse Optimization (MVO) algorithm belong to a class of nature inspired metaheuristic optimization algorithms. The algorithm, which was developed in 2015 by S. Mirjalili, 
S.M. Mirjalili and A. Hatamlou, is based on concepts of the black hole, the white hole and the worm hole. In the algorithm, the black/white hole mechanism is implemented to explore the search space while the worm hole mechanism exploits the search space.

Implementation of the black/white hole mechanism is carried out using (9).

$$
x_{i}^{j}= \begin{cases}x_{k}^{j} & r 1<N I\left(U_{i}\right) \\ x_{i}^{j} & r 1 \geq N I\left(U_{i}\right)\end{cases}
$$

where $x_{i}^{j}$ is the $j$-th parameter of the $i$-th universe (or set of decision variables), $U_{i}$ is the $i$-th universe, $N I\left(U_{i}\right)$ is the normalized inflation rate (or normalized objective function's value) of the $i$-th universe, $r 1$ is a random number (between 0 and 1) generated using MATLAB's rand command, and $x_{k}^{j}$ is the $j$-th parameter of $k$-th universe selected by a roulette wheel selection mechanism [15].

On the other hand, implementation of the worm hole mechanism is carried out using (10).

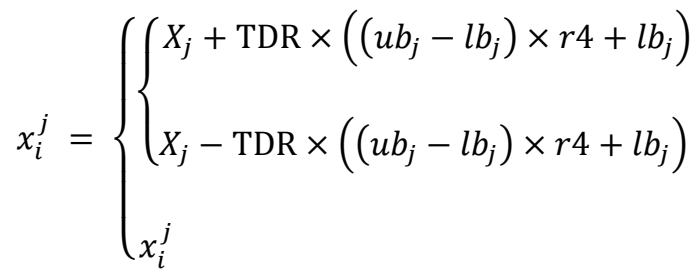

where $X_{j}$ is the $j$-th parameter of the best universe formed, Travelling Distance Rate (TDR) and Wormhole Existence Probability (WEP) are coefficients, $l b_{j}$ and $u b_{j}$ are the lower and upper bounds of the $j$-th variable; $x_{i}^{j}$ is the $j$-th parameter of the $i$-th universe and $r 2, r 3, r 4$ are random numbers (between 0 and 1). The random numbers are generated using MATLAB's rand command. Lastly, the formula for the Wormhole Existence Probability (WEP) is given by equation (11) [15].

$$
\mathrm{WEP}=\min +l \times\left(\frac{\max -\min }{L}\right)
$$

where min and max denotes minimum and maximum values respectively. The values of min and max used in this study are 0.2 and 1 respectively [15]. $l$ and $L$ are the present and maximum iterations respectively. WEP is adaptive and decreases over the iterations.

On the other hand, TDR which defines the distance rate (variation) by which an object can be teleported around the best universe obtained so far is given by equation (12) [15].

$$
\text { TDR }=1-\frac{l^{1 / p}}{L^{1 / p}}
$$

where $p$ defines the exploitation accuracy over the iterations. In this study its value was set to 6 [15]. Further, to have more precise exploitation / local search around the best universe obtained, TDR is increased over the iterations. Fig. 4 gives the general flowchart for the MVO.

\section{Proposed Cost Optimization Approach}

As earlier stated, the proposed cost optimization approach is based on MVO algorithm. MVO algorithm was adopted for use in this study after observing its effectiveness in solving several engineering optimization problems reported in [16][26]. In the cited articles, MVO algorithm-based approaches outperformed several approaches based on Flower Pollination Algorithm (FPA), Particle Swarm Optimization (PSO) algorithm, Firefly Algorithm (FFA), Gravitational Search Algorithm (GSA), Artificial Bee Colony (ABC), Fruitfly Optimization Algorithm (FOA), Differential Evolution (DE) and Ant Lion Optimizer (ALO). Further to that, Mtonga et al. [x] endeavoured to do a preliminary independent performance analysis of MVO algorithm against Chicken Swarm Optimization (CSO) algorithm, Cultural Algorithm (CA), Flower Pollination Algorithm (FPA), Gravitational Search Algorithm (GSA), and Particle Swarm Optimization (PSO) algorithm. Their study compared the six metaheuristic search algorithms on their applicability in determining optimal shunt

$$
\left.\begin{array}{l}
r 3<0.5 \\
r 3 \geq 0.5
\end{array}\right\} \begin{aligned}
& r 2<\mathrm{WEP} \\
& r 2 \geq \mathrm{WEP}
\end{aligned}
$$

capacitor sizes (to be installed at 3 predefined buses) that would minimize the total cost of active power losses and shunt capacitors' purchase in the 10-bus radial distribution system. After doing comparative simulations in MATLAB, the study found that the performance of Multiverse Optimization algorithm was better than the rest of the algorithms with which its performance was compared.

Generation of the initial search space in the proposed cost minimization approach is accomplished as follows: -

STEP 1: List all buses, except the slack bus, of the radial distribution system under consideration in a row matrix. For a radial distribution system with $m$ buses, the row matrix would be as in (13).

$$
A=[2,3, \ldots, m-1, m]
$$

The slack bus or bus number 1 is not considered as a candidate location for capacitor installation because as illustrated in Section II, the installation of shunt capacitors at this bus would not change the flow of reactive power in the branches.

STEP 2: Using MATLAB's nchoosek(A,r) command, generate the search space of buses, $B$, while taking $A$ as (13) and $r$ as being equal to 2 .

$$
B=\operatorname{nchoosek}([2,3, \ldots, m-1, m], 2)
$$

STEP 3: Create matrix $C$ which is an all zeros matrix and of the same dimensions as matrix $B$.

STEP 4: for $i=1$ : total number of rows in matrix $B$

(a) Select the $i$-th row from matrix $B$;

(b) Select the $i$-th row from matrix $C$; 


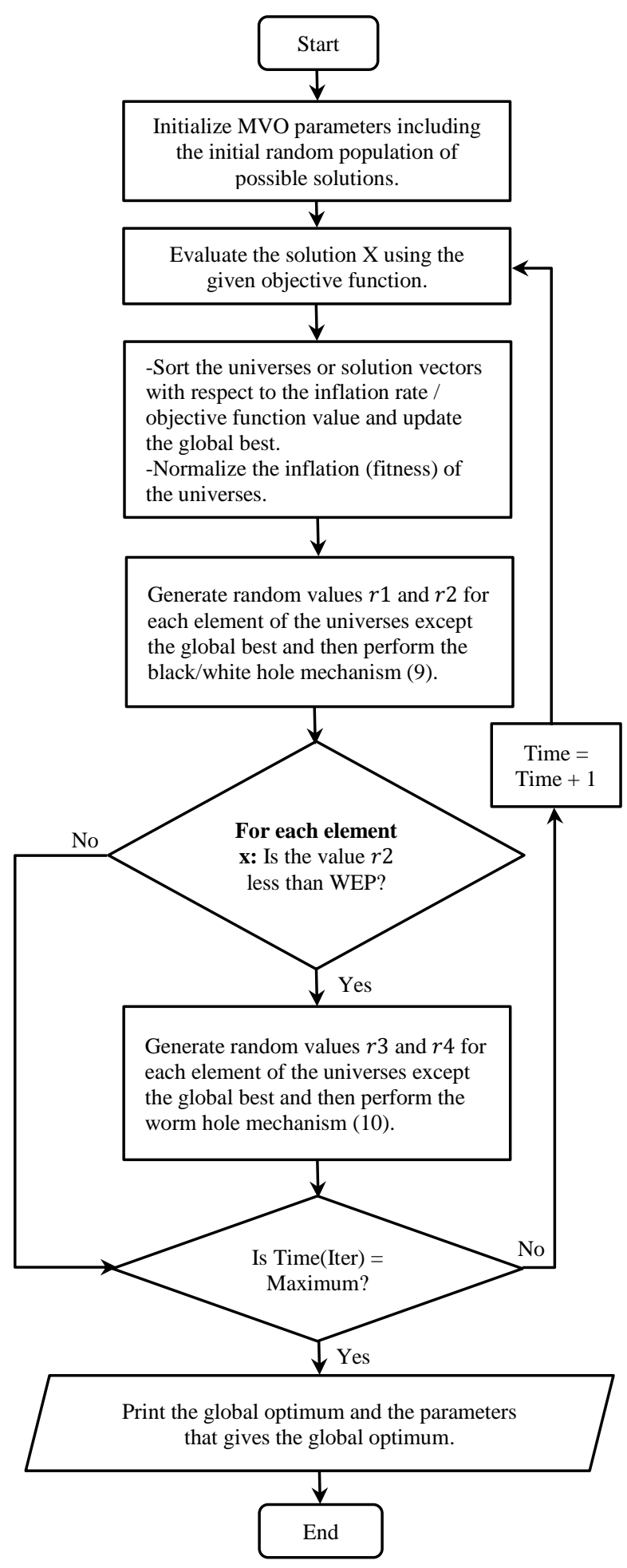

Fig. 4. Flowchart of the general steps involved in the MVO algorithm

(c) Determine the difference $|B(i, 1)-B(i, 2)|$.

(d) If $|B(i, 1)-B(i, 2)|=1$, replace the elements in the $i$-th row of matrix $C$ with the elements in the $i$-th row of matrix $B$. If $|B(i, 1)-B(i, 2)| \neq 1$, maintain the zeros in the $i$-th row of matrix $C$.

STEP 5: Use the $C(\operatorname{any}(C, 2),:)$ MATLAB command to only print out the non-zero rows of matrix $C$. Fig. 5 gives a flowchart of the preceding steps.

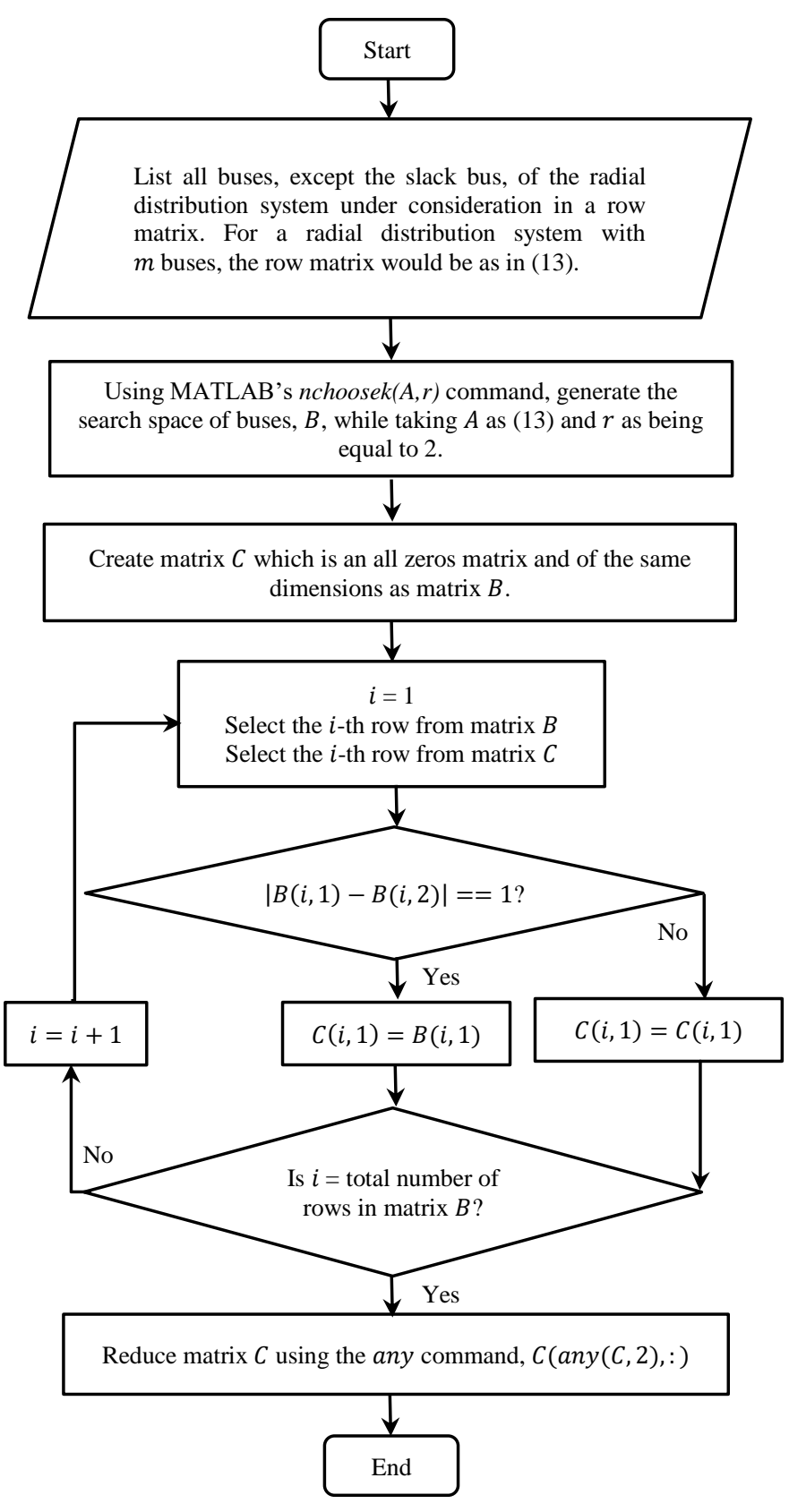

Fig. 5: Flowchart for the pairing of buses.

Thereafter, while being guided by the lower and upper limits for shunt capacitor sizes, a defined population of search agents is initialized at each position / pair, (i.e. at 2,3; 3,4 etc.), of matrix $C$. This is done in such a way that the final number of universes or set of decision variables becomes equal to the product of the defined population of search agents and the number of combinations in matrix $C$.

After initialization of the universes, the rest of the steps are as given in Fig. 4 with the exception that after implementation of the black/white hole mechanism, the lower and upper bounds are updated. Similarly, after implementation of the worm hole mechanism, the lower and upper bounds are also updated.

The lower and upper bounds are updated according to (15) 
and (16).

$$
\begin{array}{r}
l b_{i}^{j}= \begin{cases}0 & x_{i}^{j} \leq 0 \\
Q_{c n, \text { min }} & x_{i}^{j}>0\end{cases} \\
u b_{i}^{j}= \begin{cases}0 & x_{i}^{j} \leq 0 \\
Q_{c n, \max } & x_{i}^{j}>0\end{cases}
\end{array}
$$

where $l b_{i}^{j}$ and $u b_{i}^{j}$ are the $j$-th parameters of the lower and upper bounds of the $i$-th universe respectively, $Q_{c n \text {,min }}$ and $Q_{c n, \max }$ are the assumed minimum and maximum shunt capacitor sizes respectively, $x_{i}^{j}$ is the $j$-th parameter of the $i$-th universe.

The lower and upper bounds are updated to take into consideration the parameters of the universes that change after implementation of the black/white hole and worm hole mechanisms.

The number of iterations and number of search agents for MVO algorithm were set to 50 and 30 respectively [27]. On the other hand, the maximum and minimum WEP were set to 1 and 0.2 respectively [15].

\section{Test CASES: The 34- AND 85-Bus RAdial Distribution SYSTEMS}

The performance of the proposed approach was evaluated by using it to solve the optimal shunt capacitors' placement and sizing problem for cost minimization in the 34- and 85bus radial distribution systems. The following subsections discuss these two test systems.

\section{A. Test Case 1: 34-Bus Radial Distribution System.}

The system has 34 buses and 33 branches with four laterals. Its total active and reactive power loads are $4,636.50 \mathrm{~kW}$ and $2,873.50 \mathrm{kVAr}$ respectively. The rated line voltage for the system is $11 \mathrm{kV}$. Fig. 6 gives the single-line diagram for the system whereas [28] gives its line and load data.

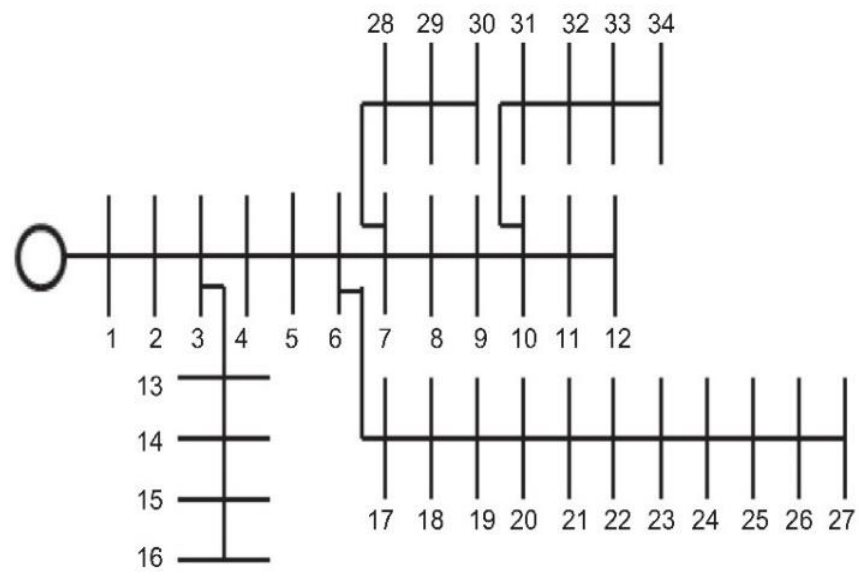

Fig. 6. Single-line diagram of the 34-bus radial distribution feeder.

\section{B. Test Case 2: 85-Bus Radial Distribution System.}

The system has 85 buses and 84 branches. Its total active and reactive power loads are 2,570.28 kW and 2,622.08 $\mathrm{kVAr}$ respectively. The rated line voltage for the system is $11 \mathrm{kV}$.
Fig. 7 gives the single-line diagram for the system whereas [29] gives its line and load data.

\section{RESULTS AND DISCUSSION}

In this section, simulation results for tests on the 34- and 85-bus radial distribution systems are presented and discussed.

\section{A. Test Case 1: The 34-Bus Radial Distribution System}

After implementing the steps given in Fig. 5, the buses of the 34-bus radial distribution system were paired as shown in matrix $C$ (17).

$$
\begin{aligned}
C= & {[2,3 ; 3,4 ; 4,5 ; 5,6 ; 6,7 ; 7,8 ; 8,9 ; 9,10 ; 10,11 ; 11,} \\
& 12 ; 12,13 ; 13,14 ; 14,15 ; 15,16 ; 16,17 ; 17,18 ; 18,19 ; \\
& 19,20 ; 20,21 ; 21,22 ; 22,23 ; 23,24 ; 24,25 ; 25,26 ; 26, \\
& 27 ; 27,28 ; 28,29 ; 29,30 ; 30,31 ; 31,32 ; 32,33 ; 33,34]
\end{aligned}
$$

which is a $(32 \times 2)$ matrix.

Thereafter, a population of 30 search agents were randomly initialized at each of the 32 pairs of buses making up matrix $C$. This resulted in the generation of 960 search agents which were then evaluated, modified and re-evaluated over 50 iterations according to the steps of the MVO algorithm. After executing the proposed approach for 100 times, the best, worst and average computation times were recorded. These are given in Table II. The results in Table II also include the net savings, power losses, voltage, optimum buses and the optimum shunt capacitor sizes. These results are further compared with those of the uncompensated case and with those that were obtained by Gravitational Search Algorithm (GSA), Cuckoo Search Algorithm (CSA), Harmony Search Algorithm (HSA), Artificial Bee Colony (ABC) algorithm and Shark Smell Optimization (SSO) algorithm based optimal shunt capacitors placement and sizing approaches.

It can be noted from Table II that the proposed approach has identified 3 as the optimum total number of buses to be compensated. Further, the proposed approach attained the least annual cost of active power losses and shunt capacitors purchase, installation, operation and maintenance than the rest of the approaches.

Fig. 8 gives a plot of bus voltage against bus number for the uncompensated and compensated cases of the 34-bus radial distribution system. It may be noted from Fig. 8 that despite elevating the bus voltages to appreciable levels, the proposed approach failed to surpass the other approaches in terms of voltage level improvement.

In addition, it may be noted from Table II that the proposed approach returns the optimal solutions with relatively high computation time than GSA, CSA, HSA, ABC and SSO based approaches. The proposed approach gives the optimum solution in about 11.9 to 20.8 minutes while the other approaches gives the optimal solution in less than a minute. Despite being relatively high, the authors still find the computation time acceptable. This is so because the installation of shunt capacitors is a power system planning issue which is to be addressed in 1 to 10 years or higher and not in seconds [9]. 


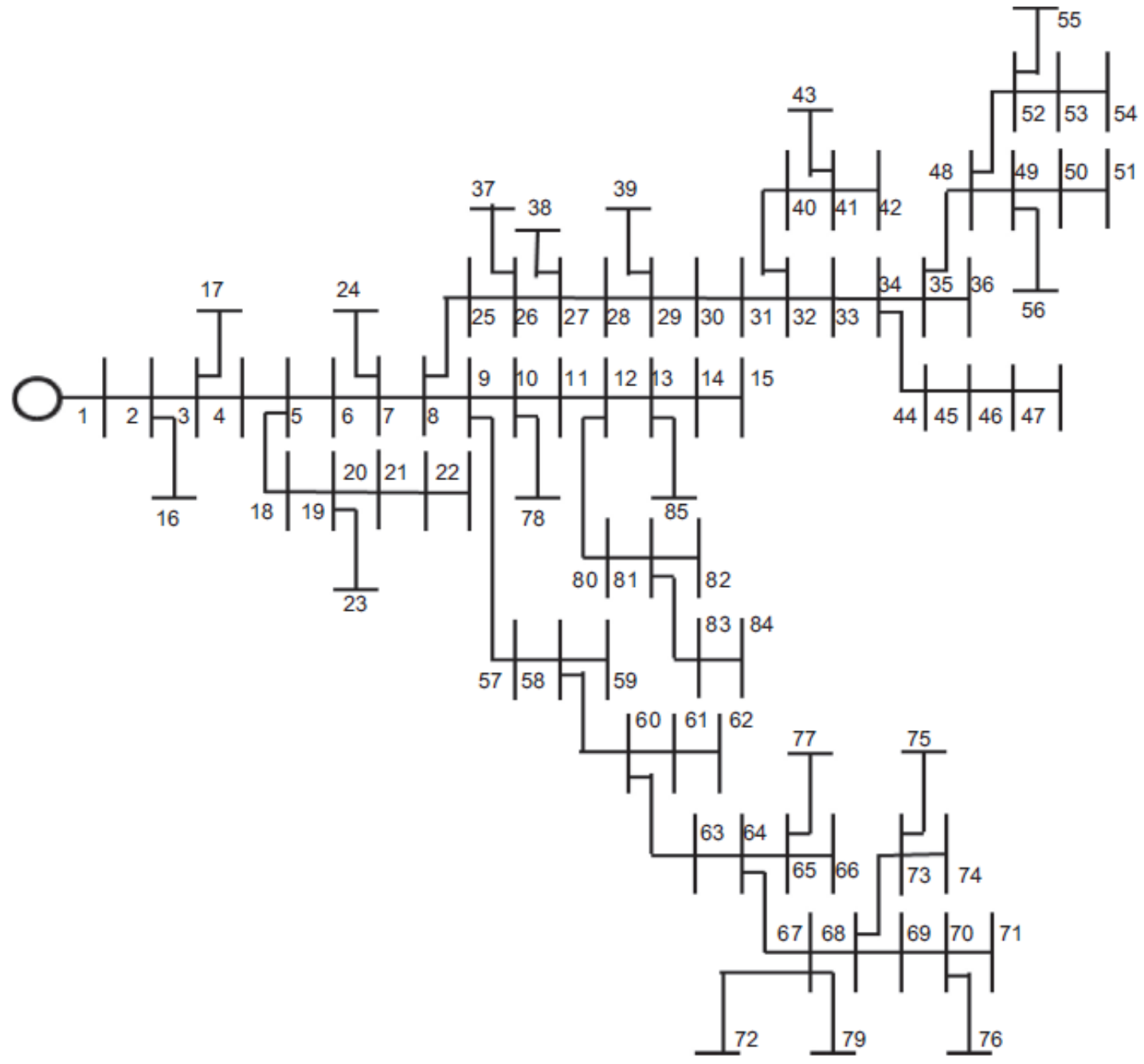

Fig. 7. Single-line diagram of the 85 -bus radial distribution feeder.

TABLE II

Comparison of Simulation Results for the 34-Bus Radial Distribution System

\begin{tabular}{|c|c|c|c|c|c|c|c|c|c|c|c|c|c|}
\hline \multirow[t]{2}{*}{ Parameters } & \multirow[t]{2}{*}{ Uncompensated } & \multicolumn{12}{|c|}{ Compensated } \\
\hline & & \multicolumn{2}{|c|}{ GSA [6] } & \multicolumn{2}{|c|}{ CSA [6] } & \multicolumn{2}{|c|}{ HSA [6] } & \multicolumn{2}{|c|}{$\mathrm{ABC}[6]$} & \multicolumn{2}{|c|}{ SSO [6] } & \multicolumn{2}{|c|}{ Proposed } \\
\hline Year & & \multicolumn{2}{|c|}{2016} & \multicolumn{2}{|c|}{2016} & \multicolumn{2}{|c|}{2016} & \multicolumn{2}{|c|}{2016} & \multicolumn{2}{|c|}{2016} & \multicolumn{2}{|c|}{2022} \\
\hline Total cost/year (\$) & $116,536.77$ & \multicolumn{2}{|c|}{$91,689.03$} & \multicolumn{2}{|c|}{$91,696.02$} & \multicolumn{2}{|c|}{$91,689.03$} & \multicolumn{2}{|c|}{$91,740.61$} & \multicolumn{2}{|c|}{$91,679.20$} & \multicolumn{2}{|c|}{$91,321.45$} \\
\hline Cost reduction/year (\%) & - & \multicolumn{2}{|c|}{21.32} & \multicolumn{2}{|c|}{21.32} & \multicolumn{2}{|c|}{21.32} & \multicolumn{2}{|c|}{21.28} & \multicolumn{2}{|c|}{21.33} & \multicolumn{2}{|c|}{21.64} \\
\hline Net savings/year (\$) & - & \multicolumn{2}{|c|}{$24,847.74$} & \multicolumn{2}{|c|}{$24,840.75$} & \multicolumn{2}{|c|}{$24,847.74$} & \multicolumn{2}{|c|}{$24,796.16$} & \multicolumn{2}{|c|}{$24,857.57$} & 25 & 15.32 \\
\hline Active power losses/hour (Kw) & 221.7214 & 160 & 4053 & 160 & 186 & 160 & 053 & 160 & 791 & 160 & 3866 & 161 & 1329 \\
\hline Loss reduction/hour (\%) & - & 27. & & 27. & & 27. & & 27.4 & & 27. & & 27. & \\
\hline Reactive power losses/hour (kVAr) & 65.1117 & 46. & & 46. & & 46.9 & & 47.1 & & 46. & 693 & 47. & 036 \\
\hline Loss reduction/hour $(\%)$ & - & 27. & & 27. & & 27.8 & & 27.6 & & 27. & & 27. & \\
\hline & & 9 & 800 & 9 & 850 & 9 & 800 & 19 & 850 & 9 & 800 & 10 & 700 \\
\hline Optimal buses and capacitor sizes & - & 19 & 850 & 19 & 850 & 19 & 850 & 24 & 750 & 19 & 900 & 20 & 750 \\
\hline & & 24 & 750 & 25 & 700 & 24 & 750 & 31 & 700 & 25 & 700 & 25 & 650 \\
\hline Total kVAr & - & 240 & & 240 & & 240 & & 230 & & 240 & & 210 & \\
\hline Annual investment cost (\$) & - & 7,3 & .00 & 7,3 & .00 & 7,38 & & 7,13 & .00 & 7,3 & 0.00 & 6,6 & 0.00 \\
\hline$V_{\min }$ (p.u.) & 0.9417 & 0.9 & & 0.9 & & 0.9 & & 0.95 & & 0.9 & & 0.9 & \\
\hline$V_{\max }$ (p.u.) & 1.0000 & 1.0 & & 1.0 & & 1.0 & & 1.00 & & 1.0 & & 1.0 & \\
\hline Best computation time (s) & - & - & & - & & - & & - & & - & & 714 & 7034 \\
\hline Worst computation time (s) & - & - & & - & & - & & - & & - & & 125 & .0905 \\
\hline Average computation time (s) & - & 16. & & 15. & & 15. & & 15.6 & & 14. & & 901 & 6299 \\
\hline
\end{tabular}




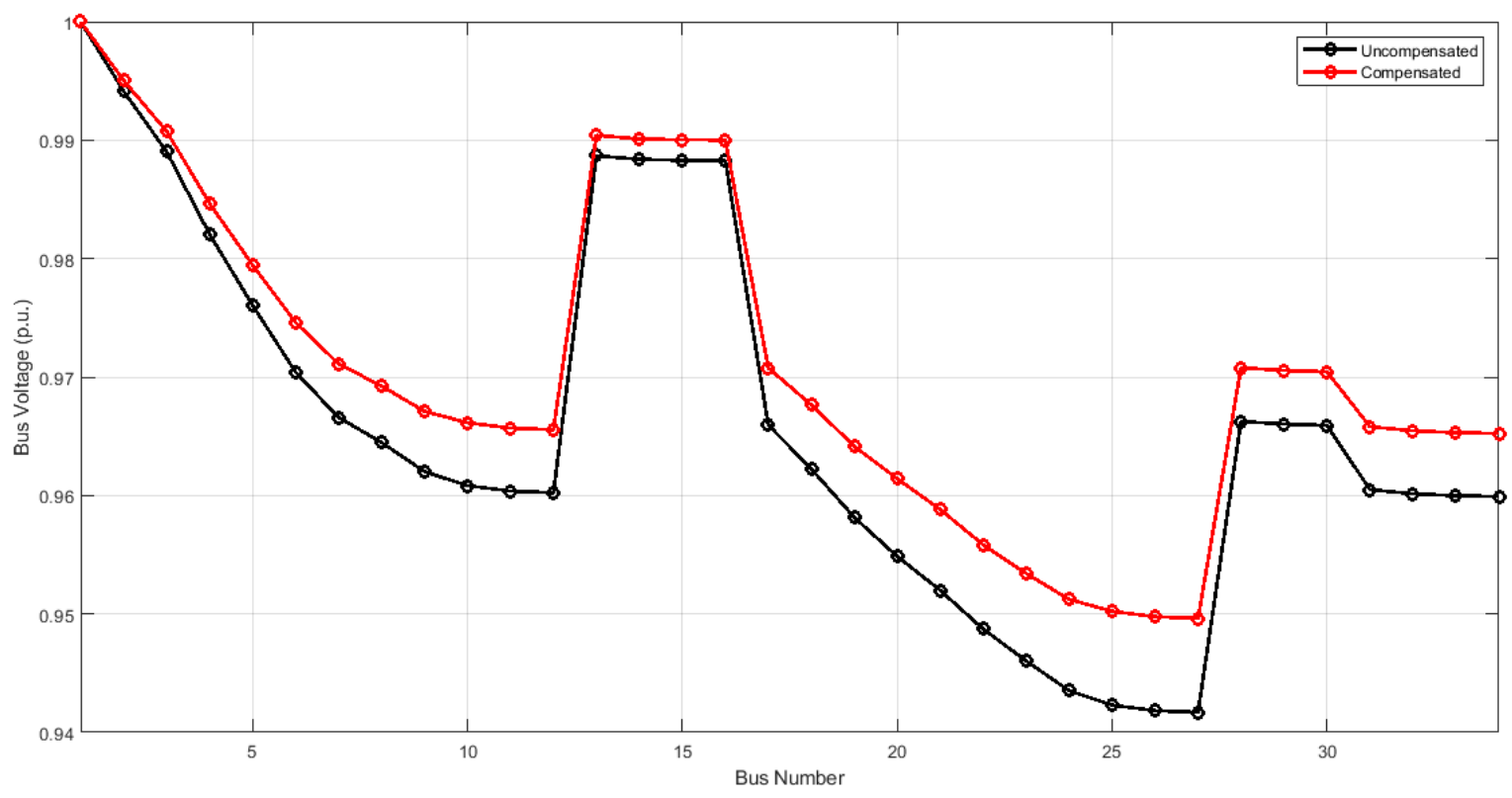

Fig. 8. Plot of bus voltage against the bus number for the 34-bus radial distribution system

\section{B. Test Case 2: The 85-Bus Radial Distribution System}

After implementing the steps given in Fig. 5 on the 85-bus radial distribution system, its buses were paired as shown in matrix $C$ (18).

$C=[2,3 ; 3,4 ; 4,5 ; 5,6 ; 6,7 ; 7,8 ; 8,9 ; 9,10 ; 10,11 ; 11$,

$12 ; 12,13 ; 13,14 ; 14,15 ; 15,16 ; 16,17 ; 17,18 ; 18,19$;

19,$20 ; 20,21 ; 21,22 ; 22,23 ; 23,24 ; 24,25 ; 25,26 ; 26$,

$27 ; 27,28 ; 28,29 ; 29,30 ; 30,31 ; 31,32 ; 32,33 ; 33,34$;

34,$35 ; 35,36 ; 36,37 ; 37,38 ; 38,39 ; 39,40 ; 40,41 ; 41$,

$42 ; 42,43 ; 43,44 ; 44,45 ; 45,46 ; 46,47 ; 47,48 ; 48,49$;

49,$50 ; 50,51 ; 51,52 ; 52,53 ; 53,54 ; 54,55 ; 55,56 ; 56$,

$57 ; 57,58 ; 58,59 ; 59,60 ; 60,61 ; 61,62 ; 62,63 ; 63,64$;

64,$65 ; 65,66 ; 66,67 ; 67,68 ; 68,69 ; 69,70 ; 70,71 ; 71$,

$72 ; 72,73 ; 73,74 ; 74,75 ; 75,76 ; 76,77 ; 77,78 ; 78,79$;

79,$80 ; 80,81 ; 81,82 ; 82,83 ; 83,84 ; 84,85$ ]

which is an $(83 \times 2)$ matrix.

Thereafter, just as it was the case with the first test system, a population of 30 search agents were also randomly initialized at each of the 83 pairs of buses making up matrix $C$. Consequently, this resulted in the generation of 2490 search agents which were then evaluated, modified and re-evaluated over 50 iterations according to the steps of the MVO algorithm. After executing the proposed approach for 100 times, the best, worst and average computation times were recorded. These are given in Table III. The results in Table III also include the net savings, power losses, voltage, optimal buses and the optimal shunt capacitor sizes. These results are further compared with those of the uncompensated case and with those that were obtained by Improved Harmony Algorithm (IHA), Sine Cosine Algorithm (SCA) and QuasiOpposition based Sine Cosine Algorithm (QOSCA) based optimal shunt capacitors' placement and sizing approaches.

It can be noted from Table III that the proposed approach gave the least annual cost of active power losses and shunt capacitors' purchase, installation, operation and maintenance. The least annual cost was attained after the installation of optimally sized shunt capacitors at 5 buses. The approaches based on SCA and QOSCA also identified 5 as the optimum total number of buses to be compensated. For these two approaches, the authors used PLI and VDI to reduce the search space of buses for the optimization process. Top 16 buses with relatively high combined values of PLI and VDI were selected as candidate buses from which SCA and QOSCA identified the optimum total number of buses and the specific optimum buses on which to install optimally sized shunt capacitors. On the other hand, the IHA based approach identified 7 as the optimum total number of buses to be compensated. For this approach the authors used LSF and VSI to reduce the search space of buses for the optimization process.

From Table III, it may further be noted that the proposed approach effectively identified the optimum buses on which to install optimally sized shunt capacitors. This was so because the proposed approach did not use any of the sensitivity-based approaches (e.g. PLI, VDI, LSF, VSI etc.) to reduce the search space. By not using any sensitivity-based approach, the proposed approach considered all the buses of the 85-bus radial distribution system as candidate buses on which optimally sized shunt capacitors would be installed. On the other hand, after the combined use of PLI and VDI, Biswal and Shankar [30] considered 16 out of the 84 buses of the 85bus radial distribution system as candidate buses for the installation of shunt capacitors. These buses are 54, 55, 51, 76, $69,74,39,72,66,28,62,38,61,60,82$ and 80 . However, it can be noted from Table III that the proposed approach identified buses 30, 48, 57, 68 and 81 as the optimum buses for the installation of shunt capacitors. These buses are not part of the top 16 buses that Biswal and Shankar [30] selected. As such it may be stated that the use of the sensitivity-based 
TABLE III

Comparison of Simulation Results for the 85-Bus Radial Distribution System

\begin{tabular}{|c|c|c|c|c|c|c|c|c|c|}
\hline \multirow[t]{2}{*}{ Parameters } & \multirow[t]{2}{*}{ Uncompensated } & \multicolumn{8}{|c|}{ Compensated } \\
\hline & & \multicolumn{2}{|c|}{ IHA [31] } & \multicolumn{2}{|c|}{ SCA [30] } & \multicolumn{2}{|c|}{ QOSCA [30] } & \multicolumn{2}{|c|}{ Proposed } \\
\hline Year & & \multicolumn{2}{|c|}{2015} & \multicolumn{2}{|c|}{2021} & \multicolumn{2}{|c|}{2021} & \multicolumn{2}{|c|}{2022} \\
\hline Total cost/year (\$) & $166,151.20$ & \multicolumn{2}{|c|}{$93,171.19$} & \multicolumn{2}{|c|}{$92,731.29$} & \multicolumn{2}{|c|}{$92,009.43$} & \multicolumn{2}{|c|}{$91,304.89$} \\
\hline Cost reduction/year (\%) & - & \multicolumn{2}{|c|}{43.92} & \multicolumn{2}{|c|}{44.19} & \multicolumn{2}{|c|}{44.62} & \multicolumn{2}{|c|}{45.05} \\
\hline Net savings/year (\$) & - & \multicolumn{2}{|c|}{$72,980.01$} & \multicolumn{2}{|c|}{$73,419.91$} & \multicolumn{2}{|c|}{$74,141.77$} & \multicolumn{2}{|c|}{$74,846.31$} \\
\hline Active power losses/hour (kW) & 316.1172 & \multicolumn{2}{|c|}{147.6050} & \multicolumn{2}{|c|}{150.5542} & \multicolumn{2}{|c|}{149.1808} & \multicolumn{2}{|c|}{148.3160} \\
\hline Loss reduction/hour (\%) & - & \multicolumn{2}{|c|}{53.31} & \multicolumn{2}{|c|}{52.37} & \multicolumn{2}{|c|}{52.81} & \multicolumn{2}{|c|}{ 53.08 } \\
\hline Reactive power losses/hour (kVAr) & 198.6019 & \multicolumn{2}{|c|}{91.8323} & 93.2 & & 92.6 & 528 & 92. & 13 \\
\hline Loss reduction/hour (\%) & - & 53.7 & & 53.0 & & 53.3 & & 53. & \\
\hline & & 8 & 250 & & & & & & \\
\hline & & 29 & 350 & 28 & 650 & 28 & 700 & 30 & 400 \\
\hline Optimal buses and capacitor sizes & - & 34 & 400 & 51 & 400 & 54 & 300 & 48 & 400 \\
\hline in $\mathrm{kVAr}$ & & 54 & 150 & 60 & 500 & 60 & 500 & 57 & 500 \\
\hline & & 58 & 350 & 66 & 200 & 69 & 250 & 68 & 450 \\
\hline & & 64 & 500 & 80 & 350 & 80 & 350 & 81 & 300 \\
\hline & & 83 & 250 & & & & & & \\
\hline Total kVAr & - & 225 & & 2100 & & 210 & & 205 & \\
\hline Annual investment cost $(\$)$ & - & 15,5 & & 13,6 & .00 & 13,6 & 00.00 & 13 , & 50.00 \\
\hline$V_{\min }$ (p.u.) & 0.8713 & 0.92 & & 0.92 & & 0.92 & & 0.9 & \\
\hline$V_{\max }$ (p.u.) & 1.0000 & 1.00 & & 1.00 & & 1.00 & & 1.0 & \\
\hline Best computation time (s) & - & 40.3 & & 7.31 & & 14.1 & & 330 & .2926 \\
\hline Worst computation time (s) & - & - & & - & & - & & 440 & 1212 \\
\hline Average computation time (s) & - & - & & - & & - & & 384 & .2046 \\
\hline
\end{tabular}

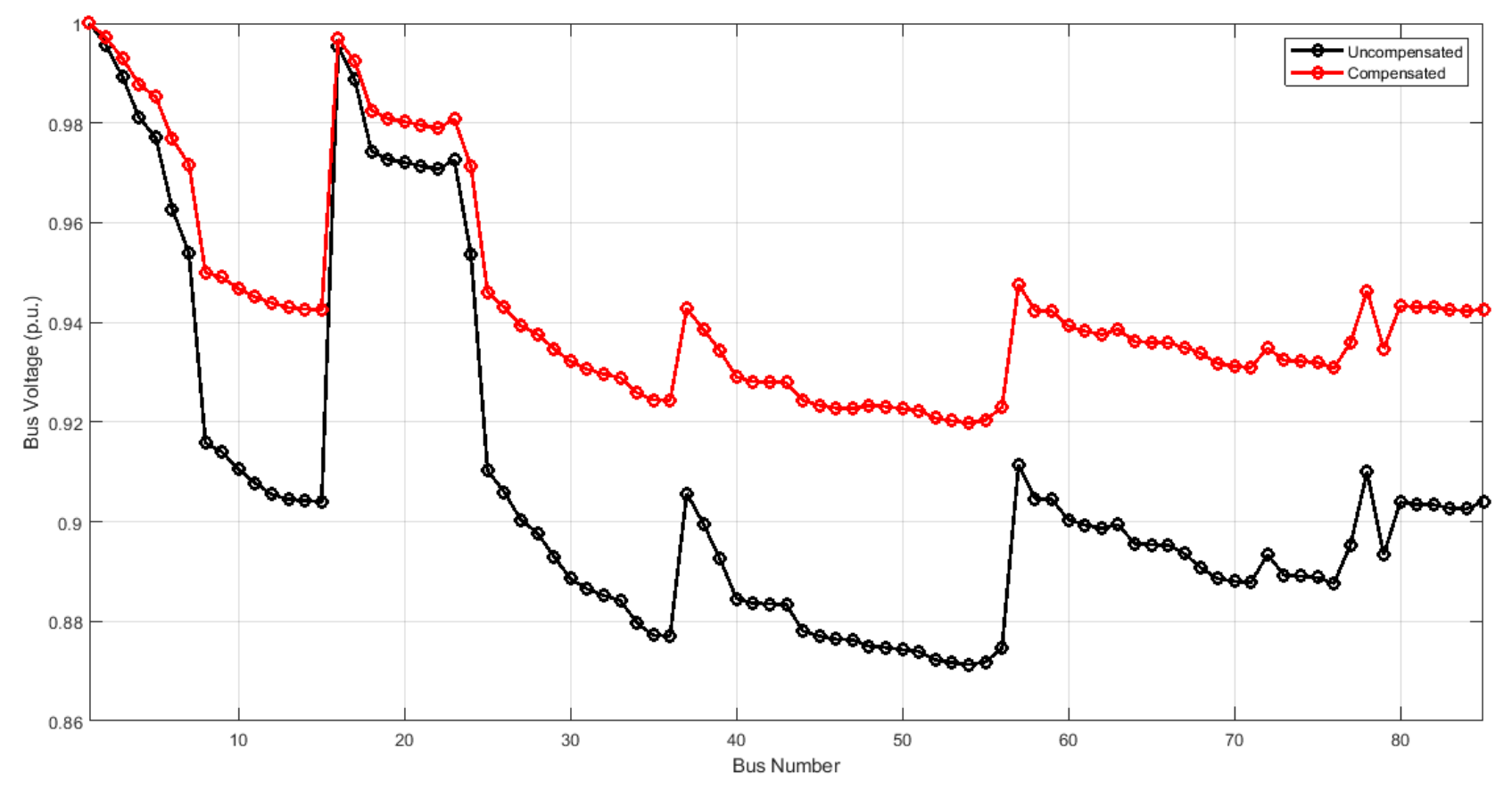

Fig. 9. Plot of bus voltage against the bus number for the 85 -bus radial distribution system

approach as well as the selection of the top 16 buses inhibits SCA and QOSCA's ability to identify the bus combination that would result in the attainment of the least annual cost of active power losses and shunt capacitors purchase, installation, operation and maintenance.

Fig. 9 gives a plot of bus voltage against bus number for the uncompensated and compensated cases of the 85-bus radial distribution system. It may be noted from Fig. 9 that despite elevating the bus voltages to appreciable levels, the proposed approach failed to surpass the other approaches in terms of voltage level improvement.

Lastly, it may be noted from Table III that the proposed approach returns the optimal solutions with relatively high computation times than IHA, SCA and QOSCA based approaches. The proposed approach gives the optimum solution in about 55.15 to 73.35 minutes while the other approaches gives the optimal solution in less than a minute. As earlier stated, despite the computation times being relatively high, the authors still find the computation time acceptable. This is so because the installation of shunt capacitors is a 
power system planning issue which is to be addressed in 1 to 10 years or higher and not in seconds [9].

\section{CONCLUSIONS}

This article has presented a novel approach for optimizing the placement and sizing of shunt capacitors in radial distribution systems with the aim of minimizing the annual cost of active power losses and shunt capacitors purchase, installation, operation and maintenance. The approach, which is based on Multiverse Optimization (MVO) algorithm, is designed to determine the optimum shunt capacitor sizes, the optimum total number of buses to be compensated and the specific optimum buses on which to install the optimally sized shunt capacitors. The proposed approach was tested on the 34and 85-bus radial distribution systems. When the simulation results were compared with those obtained by GSA, CSA, HSA, ABC, SSO, IHA, SCA and QOSCA based approaches, the developed approach stood out as a better option because it gave the least cost.

\section{REFERENCES}

[1] A. J. Wood, B. F. Wollenberg, and G. B. Sheble, "Power system security," in Power Generation, Operation, and Control, 3rd ed., Hoboken, New Jersey: John Wiley \& Sons, 2014, ch. 7, sec. 7.1, pp. 297.

[2] T. Gonen, "Application of capacitors to distribution systems," in Electric Power Distribution Engineering, 3rd ed., Boca Raton, Florida: CRC Press, 2014, ch. 8, sec. 8.5.2, pp. 455.

[3] C. Lee, H. V. H. Ayala and L. Coelho, "Capacitor placement of distribution systems using particle swarm optimization approaches," Int. J. Elect. Power Energy Syst., vol. 64, pp. 839-851, 2015.

[4] V. Haldar and N. Chakraborty, "Power loss minimization by optimal capacitor placement in radial distribution system using modified cultural algorithm," Int. Trans. Electr. Energy Syst., vol. 25, no. 1, pp. 54-71, Jan. 2015.

[5] Askarzadeh, "Capacitor placement in distribution systems for power loss reduction and voltage improvement: A new methodology," IET Gener. Transmiss. Distrib., vol. 10, no. 14, pp. 1-8, 2016.

[6] N. Gnanasekaran, S. Chandramohan, P. S. Kumar, and A. M. Imran, "Optimal placement of capacitors in radial distribution system using shark smell optimization algorithm," Ain Shams Eng. J., vol. 7, no. 2, pp. 907-916, Jun. 2016.

[7] R. M. Idris and N. M. Zaid, "Optimal shunt capacitor placement in radial distribution system," in Proc. IEEE Int. Conf. Power Energy (PECon) Nov. 2016, pp. 18-22.

[8] V. Tamilselvan, T. Jayabarathi, T. Raghunathan and X. Yang, "Optimal capacitor placement in radial distribution systems using flower pollination algorithm," Alexandria Eng. J., vol. 57, no. 4, pp. 27752786, Nov. 2018.

[9] H. Seifi and M. S. Sepasian, "Reactive power planning," in Electric power systems planning: Issues, Algorithms and Solutions, Heidelberg: Springer-Verlag, 2011, ch. 10, sec.10.4, pp. 182-184.

[10] D.P. Kothari and I.J. Nagrath (2003). Modern power system analysis (3rd ed.). New Delhi, India: McGraw Hill.

[11] R. Natarajan, "System benefits," in Power System Capacitors, Florida, USA: CRC Press, 2005, ch. 9, sec. 9.5, pp. 164.

[12] European Union, "Grid loss reduction," in Sustainable Energy Handbook. Accessed: Dec. 29, 2021. [Online]. Available: https://google.com/url?sa=t\&source=web\&rct=j\&url=https://europa.eu/c apacity4dev/file/31680/download\%3Ftoken\%3DVOPD4IAh\&ved=2ah
UKEwjLy9aRyKzpAhVdDmMBHQI3B9MQFjAAegQIBRAC\&usg=A OvVaw0i0KzOYp58qtRjSbkFIkC2

[13] A. A. El-Fergany and A. Y. Abdelaziz, "Artificial bee colony algorithm to allocate fixed and switched static shunt capacitors in radial distribution networks," Electric Power Components and Systems, vol. 42, no. 5, pp. 427-438, 2014.

[14] Voltage characteristics of electricity supplied by public electricity networks, EN Standard 50160, 2010.

[15] A S. Mirjalili, S. M. Mirjalili, and A. Hatamlou, "Multi-verse optimizer: A nature-inspired algorithm for global optimization," Neural Comput. Appl., vol. 27, no. 2, pp. 495-513, Feb. 2016.

[16] Y. T. K. Priyanto and M. Robith, "Economic dispatch and losses minimization using multi-verse optimizer on $150 \mathrm{kV}$ Mahakam transmission system," Int. J. Eng. Res. Technol., vol. 6, no. 1, pp. 489494, 2017.

[17] N. Chopra and J. Sharma, "Multi-objective optimum load dispatch using multi-verse optimization," in Proc. IEEE 1st Int. Conf. Power Electron., Intell. Control Energy Syst. (ICPEICES), Jul. 2016, pp. 1-5.

[18] I. N. Trivedi, P. Jangir, N. Jangir, S. A. Parmar, M. Bhoye, and A. Kumar, "Voltage stability enhancement and voltage deviation minimization using multi-verse optimizer algorithm," in Proc. Int. Conf. Circuit, Power Comput. Technol. (ICCPCT), Mar. 2016, pp. 1-5.

[19] B. Bentouati, S. Chettih, P. Jangir, and I. N. Trivedi, "A solution to the optimal power flow using multi-verse optimizer," J. Electr. Syst., vol. 12, no. 4, pp. 716-733, 2016.

[20] D. Guha, P. K. Roy, and S. Banerjee, "Multi-verse optimisation: A novel method for solution of load frequency control problem in power system," IET Gener., Transmiss. Distrib., vol. 11, no. 14, pp. 36013611, Sep. 2017.

[21] H. Zhao, X. Han, and S. Guo, "DGM $(1,1)$ model optimized by MVO (multi-verse optimizer) for annual peak load forecasting," Neural Comput. Appl., vol. 30, no. 6, pp. 1811-1825, Sep. 2018.

[22] A. Fathy and H. Rezk, "Multi-verse optimizer for identifying the optimal parameters of PEMFC model," Energy, vol. 143, pp. 634-644, Jan. 2018.

[23] a M. A. Elfattah, A. E. Hassanien, S. Abuelenin, and S. Bhattacharyya, "Multi-verse optimization clustering algorithm for binarization of handwritten documents," in Recent Trends in Signal and Image Processing (Advances in Intelligent Systems and Computing), vol. 727. Singapore: Springer, 2019, pp. 165-175.

[24] H. Singh, S. Mehta, and S. Prashar, "Economic load dispatch using multi verse optimization," Int. J. Eng. Res., vol. 2, no. 6, pp. 43-51, 2016.

[25] A. Kumar and S. Suhag, "Multiverse optimized fuzzy-PID controller with a derivative filter for load frequency control of multisource hydrothermal power system," TURKISH J. Electr. Eng. Comput. Sci., vol. 25, no. 5, pp. 4187-4199, 2017.

[26] A. Kumar and S. Suhag, "Effect of TCPS, SMES, and DFIG on load frequency control of a multi-area multi-source power system using multi-verse optimized fuzzy-PID controller with derivative filter," $J$. Vib. Control, vol. 24, no. 24, pp. 5922-5937, Dec. 2018.

[27] T.P.M. Mtonga, K.K. Kaberere, and G.K. Irungu, "Performance analysis of six search algorithms in minimizing the cost of real power losses and shunt capacitors purchase through optimal shunt capacitors sizing," in Proceedings of the 2020 Sustainable Research and Innovation (SRI) Conference, (pp. 130-143), JKUAT Main Campus, Kenya.

[28] M. Chis, M. M. A. Salama and S. Jayaram, "Capacitor placement in distribution systems using heuristic search strategies," IEE Proceedings - Generation, Transmission and Distribution, vol. 144, pp. 225-230, 1997.

[29] D.B. Prakash and C. Lakshminarayana, "Optimal siting of capacitors in radial distribution network using Whale Optimization Algorithm," Alexandria Eng. J., vol. 56, no. 4, pp. 1-11, Nov., 2016.

[30] S. R. Biswal and G. Shankar, "A novel quasi-opposition-based sine cosine algorithm for optimal allocation and sizing of capacitor in radial distribution systems," [Preprint] November, 2021, [accessed: 14 ${ }^{\text {th }}$ December, 2021]. DOI: 10.21203/rs.3.rs-1070297/v1 